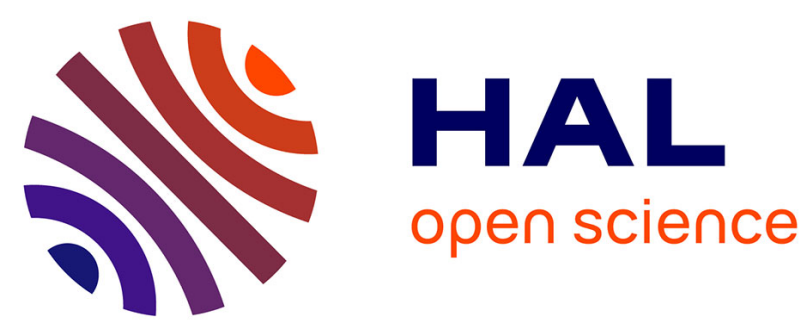

\title{
Trans-1,2-disiloxybenzocyclobutene, an adequate partner for the auto-oxisation: EPR/spin trapping and theoretical studies
}

\author{
Jean Drujon, Raphaël Rahmani, Virginie Héran, Romain Blanc, Yannick \\ Carissan, Béatrice Tuccio, Laurent Commeiras, Jean-Luc Parrain
}

\section{To cite this version:}

Jean Drujon, Raphaël Rahmani, Virginie Héran, Romain Blanc, Yannick Carissan, et al.. Trans-1,2disiloxybenzocyclobutene, an adequate partner for the auto-oxisation: EPR/spin trapping and theoretical studies. Physical Chemistry Chemical Physics, 2014, 16, pp.7513-7520. 10.1039/c3cp55077j . hal-01066302

\section{HAL Id: hal-01066302 https://hal.science/hal-01066302}

Submitted on 19 Sep 2014

HAL is a multi-disciplinary open access archive for the deposit and dissemination of scientific research documents, whether they are published or not. The documents may come from teaching and research institutions in France or abroad, or from public or private research centers.
L'archive ouverte pluridisciplinaire HAL, est destinée au dépôt et à la diffusion de documents scientifiques de niveau recherche, publiés ou non, émanant des établissements d'enseignement et de recherche français ou étrangers, des laboratoires publics ou privés. 


\section{PAPER}

Cite this: Phys. Chem. Chem. Phys., 2014, 16, 7513

Received 2nd December 2013, Accepted 13th February 2014

DOI: $10.1039 / c 3 c p 55077 j$

www.rsc.org/pccp

\section{trans-1,2-Disiloxybenzocyclobutene, an adequate partner for the auto-oxidation: EPR/spin trapping and theoretical studies $\dagger$}

\author{
Jean Drujon, ${ }^{a}$ Raphaël Rahmani, ${ }^{a}$ Virginie Héran, ${ }^{a}$ Romain Blanc, ${ }^{a}$ \\ Yannick Carissan, ${ }^{\star a}$ Béatrice Tuccio, ${ }^{b}$ Laurent Commeiras*a and Jean-Luc Parrain ${ }^{\star a}$
}

The auto-oxidation of trans-1,2-disiloxybenzocyclobutene 1 was found to be very efficient, giving endoperoxide $\mathbf{7}$ in quantitative yield. Each step of the mechanism of spin-forbidden addition of triplet oxygen $\mathrm{O}_{2}\left({ }^{3} \Sigma_{\mathrm{g}}\right)$ was studied by both EPR/spin trapping and theoretical studies.

\section{Introduction}

For several years now, the preparation and the reactivity of $\gamma$-alkylidenebutenolides have been studied in our laboratory in order to exploit their intrinsic features. ${ }^{1}$ Among the directions taken into account, we have used these building blocks to design fused polycyclic spirolactones ${ }^{2}$ in a step-economical fashion. Because cycloaddition reactions are among the most useful processes for generating molecular complexity, inter- and intramolecular [4+2]-cycloaddition reactions between trans-1,2disiloxybenzocyclobutenes 1 and $\delta$-substituted $\gamma$-alkylidenebutenolides $\mathbf{2}$ yielded, under very mild conditions, these spirolactone moieties $\mathbf{3}$ or naphthofuranone moieties $\mathbf{4}$ and, at least, gave an access to deoxylambertellol $\mathrm{B} \mathbf{5}^{3}$ and the CDEF ring system of lactonamycinone 6 (Scheme 1). ${ }^{4}$ During these studies, we observed that trans-1,2-disiloxybenzocyclobutene $\mathbf{1}$ was very reactive towards the dioxygen, even without any activation, giving the corresponding endo-peroxide 7 which has been detected during both preparation of $\mathbf{1}$ (reduction of diketone $\mathbf{8}$ followed by protection of the hydroxyl function) and in subsequent [4+2]-cycloaddition reaction. Particularly, we found that the use of oxygen free solvents under strictly inert Ar atmosphere was a crucial point to obtain good yields in the preparation of 3 or 4 . Without paying attention to this last point, up to $40 \%$ of stable endo-peroxide 7 was for example observed in the crude ${ }^{1} \mathrm{H}$ NMR (Scheme 1).

In spite of the numerous uses of disilyloxybenzocyclobutene 1 or derivatives by several groups in Diels-Alder reaction, ${ }^{5}$ no

\footnotetext{
${ }^{a}$ Aix Marseille Université, Centrale Marseille, CNRS, iSm2 UMR 7313, 13397, Marseille, France.E-mail: yannick.carissan@univ-amu.fr, laurent.commeiras@univ-amu.fr, jl.parain@univ-amu.fr; Fax: +33-491-289-187; Tel: $+33-491-289-126$

${ }^{b}$ Aix Marseille Université, CNRS, ICR, UMR 7273, 13397 Marseille, France $\dagger$ Electronic supplementary information (ESI) available: Synthesis procedure, ${ }^{1} \mathrm{H}$ and ${ }^{13} \mathrm{C}$ NMR spectra, computational details, and the UV spectrum of $\mathbf{1}$ at $400 \mathrm{~nm}$. See DOI: $10.1039 / \mathrm{c} 3 \mathrm{cp} 55077 \mathrm{j}$
}

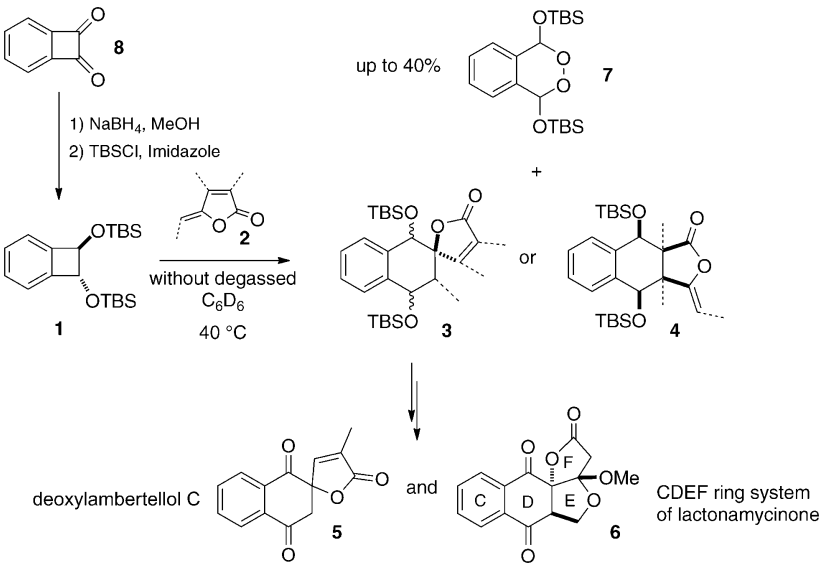

Scheme 1 Synthesis of CDEF ring system of lactonamycinone.

one has described this interesting reactivity. ${ }^{6}$ Moreover, to the best of our knowledge, only one example, using drastic conditions $\left(T=190-240{ }^{\circ} \mathrm{C}\right)$, was reported for the trapping of dioxygen by benzocyclobutene. ${ }^{7}$ In our case, the reaction was performed at lower temperature $\left(40{ }^{\circ} \mathrm{C}\right)$ and within 6 hours. Reaction times can also dramatically decrease using a saturated atmosphere of dioxygen (Scheme 2).

This oxidation process is the major source of material degradation and the most important problem of the shelf-life of organic compounds such as polymers, lipids or living organisms... Actually, two processes have been assigned for
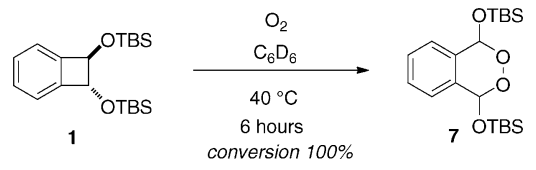

Scheme 2 Reaction of 1 with dioxygen. 
this transformation. The first one, which is a rapid process, involved singlet oxygen $\mathrm{O}_{2}\left({ }^{1} \Sigma_{\mathrm{g}}\right)$ generally obtained via a photocatalytic activation; the second one, slower than the first one, involved triplet oxygen $\mathrm{O}_{2}\left({ }^{3} \Sigma_{\mathrm{g}}\right)$ without any activation, often called auto-oxidation or "dark" oxidation. In some cases, and with a particular type of olefins, the auto-oxidation can occur rapidly. The ease of benzocyclobutene $\mathbf{1}$ for auto-oxidation to give endo-peroxide 7 fits into this pattern and led us to study the mechanism of this reaction by both EPR/spin trapping and computational approach.

As a possible scenario (Scheme 3), the following mechanistic pathway could be proposed. First of all, the disrotatory opening of 1 could lead to the formation of $o$-quinodimethane $\mathbf{9}$. The spinforbidden addition of triplet oxygen $\mathrm{O}_{2}\left({ }^{3} \Sigma_{\mathrm{g}}\right)$ to 9 would furnish triplet $\mathbf{1 0}$ and finally the singlet ground state endo-peroxide 7. It is worthy of note that the change in multiplicity of biradical 10 from a triplet to a singlet is necessary to give 7 and should be proven by calculation.

As described in Scheme 4, conventional electron paramagnetic resonance (EPR) spectroscopy associated with the spin-trapping technique is the tool of choice to study fleeting radical intermediates. In this context, the fast addition of a radical to a diamagnetic spin-trap (usually a nitrone or a nitroso compound) leads to a longlived paramagnetic spin adduct (a nitroxide) that can be easily detected by EPR spectroscopy ${ }^{8}$ and can give much information about the addend structure. So, the spin trapping using the commercially available traps (2-methyl-2-nitrosopropane (MNP) 11 and the ${ }^{31} \mathrm{P}$-labelled nitrone diethyl(2-methyl-1-oxido-3,4-dihydro2H-pyrrol-2-yl)phosphonate (DEPMPO) 12), followed by analysis by EPR spectroscopy should confirm the transient existence of the triplet biradical $10\left({ }^{3} \Sigma_{\mathrm{g}}\right)$ formed during the auto-oxidation process.

To complete the experimental studies of the reaction, we also performed a complete theoretical study of the reaction pathway. In particular, we focused on the inter system crossing (ISC) which explains why $\mathrm{O}_{2}\left({ }^{3} \Sigma_{\mathrm{g}}\right)$ could react with a singlet molecule with no activation, as normally required. ${ }^{9}$ In this study, we have shown that 9 exists and could react with $\mathrm{O}_{2}\left({ }^{3} \Sigma_{\mathrm{g}}\right)$, which leads to

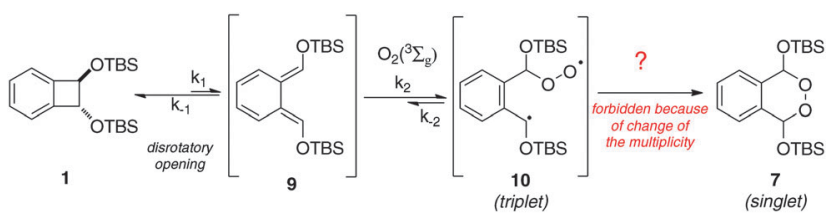

Scheme 3 Proposal mechanism.

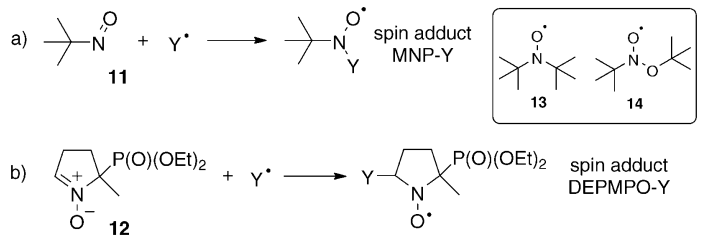

Scheme 4 Spin trapping of a radical $Y^{\bullet}$ using (a) the nitroso compound MNP 11 or (b) the nitrone DEPMPO 12, and structures of the nitroxide spin adducts formed. an oxygen containing biradical molecule, 10. This latter triplet molecule should undergo an ISC due to spin orbit coupling and should lead to the singlet product $7 .{ }^{10}$ We also suggested the possibility of a light activated pathway, which implies an ISC between excited states of 9 .

\section{Results and discussion}

\section{Spin trapping experiments ${ }^{11}$}

In a preliminary experiment and to confirm the utility of the spin trapping technique, we verified that no EPR signal could be observed from a solution of $\mathbf{1}$ (in the absence of spin traps $\mathbf{1 1}$ or 12) in benzene, with or without dioxygen, whatever the reaction time. Interestingly the two spin traps used in our study exhibit different features. Spin adducts obtained after addition of carboncentered radicals on a nitroso trap ${ }^{12}$ are usually stable enough to be EPR-observed while nitroso-derived adducts obtained from oxygen-centered radicals are almost impossible to detect due to thermal and photochemical instability. ${ }^{13,14}$

In a first step, a solution containing MNP $11\left(0.2 \mathrm{~mol} \mathrm{~L}^{-1}\right)$ and $1\left(0.2 \mathrm{~mol} \mathrm{~L}^{-1}\right)$ was prepared in benzene. After $c a .2 \mathrm{~h}$ at $40{ }^{\circ} \mathrm{C}$, oxygen was removed by argon bubbling before recording the EPR spectrum of the medium. Its simulation performed using the WinSim software ${ }^{15}$ revealed the presence of three different paramagnetic species. The first $\left(a_{\mathrm{N}}=1.54 \mathrm{mT}\right)$ and second $\left(a_{\mathrm{N}}=2.63 \mathrm{mT}\right)$ signals were also observed in blank tests performed in the absence of $\mathbf{1}$. According to data previously published, ${ }^{16}$ the above-mentioned signals were, respectively, assigned to radicals $\mathbf{1 3}$ and $\mathbf{1 4}$ (Scheme 4) that correspond to MNP decomposition products formed upon light exposure of the spin trap. The third detected nitroxide 15, which was never observed in the absence of either 1 or $\mathrm{O}_{2}$, showed a six line EPR spectrum $\left(a_{\mathrm{N}}=1.44 \mathrm{mT}\right.$ and $\left.a_{\mathrm{H}}=0.13 \mathrm{mT}\right)$. The spin adduct 15 was thus formed after reaction of dioxygen with benzocyclobutene $\mathbf{1}$, and obviously resulted from the trapping of a radical centered on a secondary carbon, as indicated by the presence of a small hyperfine coupling with one hydrogen nucleus.

In a second step, phosphorylated nitrone DEPMPO 12 (reported to yield persistent spin adducts with oxygen-centered radicals) assisted spin trapping assays were conducted. ${ }^{17}$ As for MNP experiments, solutions missing either 1 or dioxygen were found to be EPR silent. Heating $\left(40{ }^{\circ} \mathrm{C}\right)$ an oxygenated benzene solution containing $1\left(0.1 \mathrm{~mol} \mathrm{~L}^{-1}\right)$ and DEPMPO $12\left(0.1 \mathrm{~mol} \mathrm{~L}^{-1}\right)$ for 10 min gave rise to the EPR spectrum given in Fig. 1, recorded after dioxygen removal.

A signal including 24 lines due to hyperfine coupling reactions with nitrogen, phosphorus, $\beta$-hydrogen and $\gamma$-hydrogen nuclei $\left(a_{\mathrm{N}}=1.27 \mathrm{mT}, a_{\mathrm{P}}=3.84 \mathrm{mT}, a_{\mathrm{H} \beta}=0.73 \mathrm{mT}\right.$ and $a_{\mathrm{H} \gamma}=$ $0.18 \mathrm{mT}$ ) was recorded. The first integration of this signal was checked and did not lead to a bell-shaped curve characteristic of the presence of a strong electron exchange coupling. In addition, the EPR lines were not broadened by an electron spin-electron spin dipolar interaction. This clearly shows that the EPR spectrum recorded corresponded to a monoradical nitroxide. Though the existence of a long distance coupling 


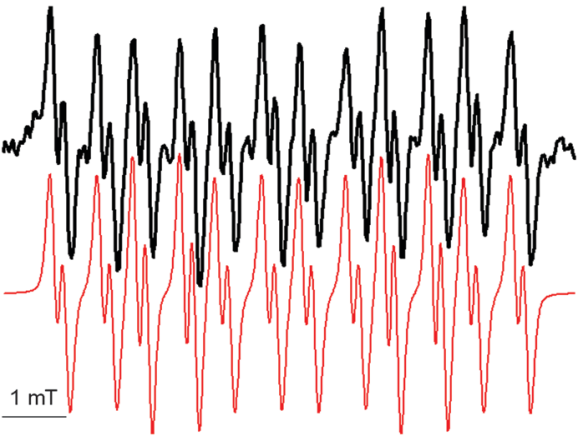

Fig. 1 Experimental EPR signal of $\mathbf{1 6}$ (black full lines) obtained in benzene after 10 min of reaction at $40{ }^{\circ} \mathrm{C}$ between dioxygen and $1\left(0.1 \mathrm{~mol} \mathrm{~L}^{-1}\right)$ in the presence of DEPMPO $12\left(0.1 \mathrm{~mol} \mathrm{~L}^{-1}\right)$. Dioxygen was removed by argon bubbling before recording the spectrum in order to observe narrower lines. The simulation (red lines) led to the following parameters: $a_{\mathrm{N}}=1.27 \mathrm{mT}, a_{\mathrm{P}}=3.84 \mathrm{mT}, a_{\mathrm{H} \beta}=0.73 \mathrm{mT}$ and $a_{\mathrm{H} \gamma}=0.18 \mathrm{mT}$.

with a $\gamma$-hydrogen atom is rather uncommon, it is usually observed in the EPR spectra of hydroperoxyl or alkylperoxyl radical adducts of five-membered cyclic nitrones. After a comparison of the results thus obtained with DEPMPO 12 with literature data, a likely hypothesis is to consider that this species corresponds to an alkylperoxyl radical adduct 16 of DEPMPO. ${ }^{17 c, 18}$ This implies that, after the addition of the peroxy radical function of $\mathbf{1 0}$ to DEPMPO, the carbon-centered radical moiety rapidly evolved, but we are not able to describe the exact structure of the monoradical thus formed since EPR spectroscopy can only bring structural information in the vicinity of the paramagnetic center. At this stage, one could envisage the possibility that the oxygencentered radical trapped by DEPMPO could result from the decomposition of 7. To discard this hypothesis, control spin trapping experiments were performed starting from endoperoxide 7. An oxygenated solution containing DEPMPO $\left(0.1 \mathrm{~mol} \mathrm{~L}^{-1}\right)$ and $7\left(0.1 \mathrm{~mol} \mathrm{~L}^{-1}\right)$ was prepared in benzene. An EPR spectrum recorded after $c a .1 \mathrm{~h}$ (and after oxygen removal) showed no signal. The same experiment was performed in the presence of trace metal ions (added by letting the solution go through an iron needle). Whether the reaction was performed in the presence of oxygen or not, a 12-line EPR signal was systematically recorded. Comparing the hyperfine coupling constant values determined after its simulation $\left(a_{\mathrm{N}}=1.24 \mathrm{mT}, a_{\mathrm{P}}=\right.$ $4.70 \mathrm{mT}$, and $a_{\mathrm{H} \beta}=0.77 \mathrm{mT}$ ) to previously published data led to assign the signal to a sterically hindered alkoxyl radical spin adduct. These results proved two points. (i) In the presence of metal traces, a ring opening could be observed in endoperoxide 7, which yielded an alkoxyl radical; this reaction did not require the presence of dioxygen. (ii) The DEPMPO spin adduct detected starting from $\mathbf{1}$ did not come from the decomposition of the oxidation product 7 eventually present in the medium.

All the experiments described were repeated twice, and identical results were obtained. It is also worthy of mention that, in general, the radical trapping by a nitrone is a kinetically slow reaction, all the more so since the radical is sterically hindered, while the reactions performed with nitroso spin traps are usually more rapid. ${ }^{19}$ Thus, a radical centered on a secondary

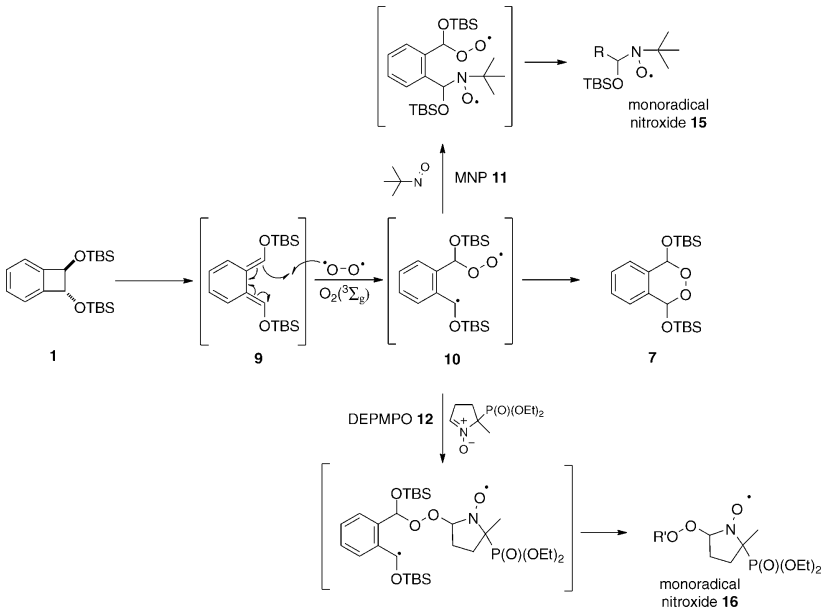

Scheme 5 EPR/Spin trapping experiments.

carbon could react much faster with dioxygen, yielding a peroxyl radical, than with DEPMPO. These kinetic reasons could explain why no carbon-centered radical adduct of DEPMPO was EPRdetected in our experiments. This study brings evidence that the auto-oxidation reaction of $\mathbf{1}$ could likely follow a radical pathway (Scheme 5). Although EPR alone is not sufficient to establish precisely the mechanism involved, the presence of free radical intermediates was undoubtedly proven. A MNP adduct of a radical centered on a secondary carbon was EPR-observed, while a peroxyl radical was detected using the nitrone DEPMPO 12. Both adducts could be formed after trapping the triplet intermediate 10, the biradical adducts initially formed probably evolved to the monoradical nitroxide species detected. ${ }^{20}$

At this stage, though the spin trapping experiments were consistent with the proposed mechanism, some points remain unclear as for example the very high yield of the process. So in order to acquire supplemental information, theoretical studies were undertaken to confirm the proposal mechanism, the EPR/spin trapping results and, more particularly, the change in multiplicity of biradical $\mathbf{1 0}$ from a triplet to a singlet ground state. Each step is detailed in the following paragraphs.

\section{Theoretical study}

2.1. Computational details. The study of the reaction pathway was carried out by determining the stationary points and related energy differences. In order to reduce the computational time, TBS groups were modelled by TMS. The geometrical structure optimizations were performed using density functional theory (DFT) with the PBE0 ${ }^{21}$ functional. The def2-TZVP basis set $^{22,23}$ was used on all atoms. The integration grid used was $\mathrm{m} 3$ as defined in the TURBOMOLE program package and the energy threshold was set to $1 \mathrm{~d}^{-6}$ atomic units. Unless otherwise specified, all DFT singlet densities (respectively triplet) were obtained in a restricted (respectively unrestricted) formalism. All minima on the energy potential surface were characterized by frequency calculations, all of them being positive.

All transition states exhibit one and only one negative frequency, which is along the reaction coordinate. The excited 
state calculations along the opening of the $\mathrm{C}_{4}$ ring in $\mathbf{1}$ and for the UV spectrum were done using the $\mathrm{CC} 2{ }^{24}$ method with the aug-cc-pVDZ basis sets ${ }^{25}$ and related auxiliary basis sets. ${ }^{26}$ Along this opening, the molecule keeps a symmetry axis of order 2. Thus, all calculations along this pathway were done in the $C_{2}$ point symmetry group. Configuration Interaction with Single and Double excitations $(\mathrm{CISD})^{27,28}$ calculations were performed with the def2-TZVP basis set on PBE0/def2-TZVP optimized geometries. Eight electrons were correlated. DFT energies contain the zero point energy corrections. This ZPE correction does not influence drastically the energy differences and could have been ignored. Thus, the CISD calculations were left uncorrected. DFT optimizations and CC2 calculations were carried out using the TURBOMOLE 6.3 program package ${ }^{29}$ and CISD calculations were performed by the use of the MOLPRO program package. ${ }^{30}$ Basis sets for MOLPRO were obtained via the EMSL website. ${ }^{31}$ Geometries are provided as ESI. $\dagger$ A scaling factor of $0.9593^{32}$ was used to compute thermodynamical properties.

2.2. Disrotatory opening of the $\mathrm{C}_{4}$ ring: proof of existence of 9 by $\mathrm{UV} /$ visible analysis. The disrotatory opening of the $\mathrm{C}_{4}$

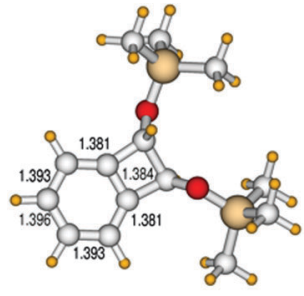

1

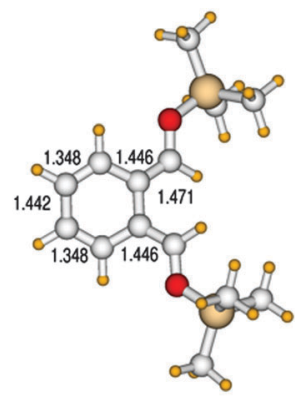

9
Fig. 2 Optimized geometries of $\mathbf{1}$ and $\mathbf{9}$. Indicated bond distances are in Angstrom. ring of TMS-1 is a kinetically relevant step of the reaction (Fig. 7). The activation energy of this reaction, calculated as the energy difference between TS $_{1-9}$ and TMS-1, is $26.8 \mathrm{kcal} \mathrm{mol}^{-1}$. The reaction energy i.e., the energy difference between TMS-9 and TMS-1, is $10.6 \mathrm{kcal} \mathrm{mol}^{-1}$. The $\mathrm{C}-\mathrm{C}$ distances in the $\mathrm{C}_{6}$ ring (Fig. 2) show a loss of aromaticity in TMS-9: two of them are short $\mathrm{C}_{3}-\mathrm{C}_{4}$ and $\mathrm{C}_{5}-\mathrm{C}_{6}(1.35 \AA)$, and four of them are long, around $1.45 \AA$, and can be considered as single bonds ( $\mathrm{C}-\mathrm{C}$ bond distances are $1.54 \AA$ in hexane). This explains the destabilization of TMS-9 with respect to TMS-1.

At room temperature, as was already mentioned by Danishefsky and Choy, ${ }^{5 e, 33}$ benzocyclobutene 1 , exhibits a light yellow colour. It is worthy of note that the yellow colour disappears at low temperature. This thermochromism could be explained by an inspection of the potential energy surfaces of the ground and the first excited states of the molecule along the reaction coordinate corresponding to the opening of the $\mathrm{C}_{4}$ ring (Fig. 3). At low temperature, the crossing of the barrier is not possible and certainly only $\mathbf{1}$ is present in the medium. As can be seen in Fig. 3, the energy difference between the ground and the first excited states is roughly $5.19 \mathrm{eV}$.

Such a radiation is in the far ultraviolet $(239 \mathrm{~nm})$. When the temperature is high enough to cross the barrier, TMS-9 is formed and absorbs at around $400 \mathrm{~nm}$ in a broad range as the UV/visible spectrum shows. This corresponds to violet. The complementary colour of violet, yellow, is then observed.

We have performed the UV/visible spectrum of the reactant. We want to determine whether 1 exists alone or in equilibrium with 9. Furthermore, as the reaction with $\mathrm{O}_{2}$ is easy, we compare the experimental UV/visible spectrum to the ones computed for TMS-1, TMS-9 and TMS-7 (Fig. 4). In this spectrum, a shoulder at $330 \mathrm{~nm}$ and a weak absorption at $400 \mathrm{~nm}$ are visible. According to calculations (Table 1 and Fig. 4), only TMS-9 is likely to absorb at wavelengths larger than $300 \mathrm{~nm}$ at two wavelengths compatible with experiment $(324 \mathrm{~nm}$ for $330 \mathrm{~nm}$ and $394 \mathrm{~nm}$ for $400 \mathrm{~nm}$ ). Furthermore, we have detected phosphorescence
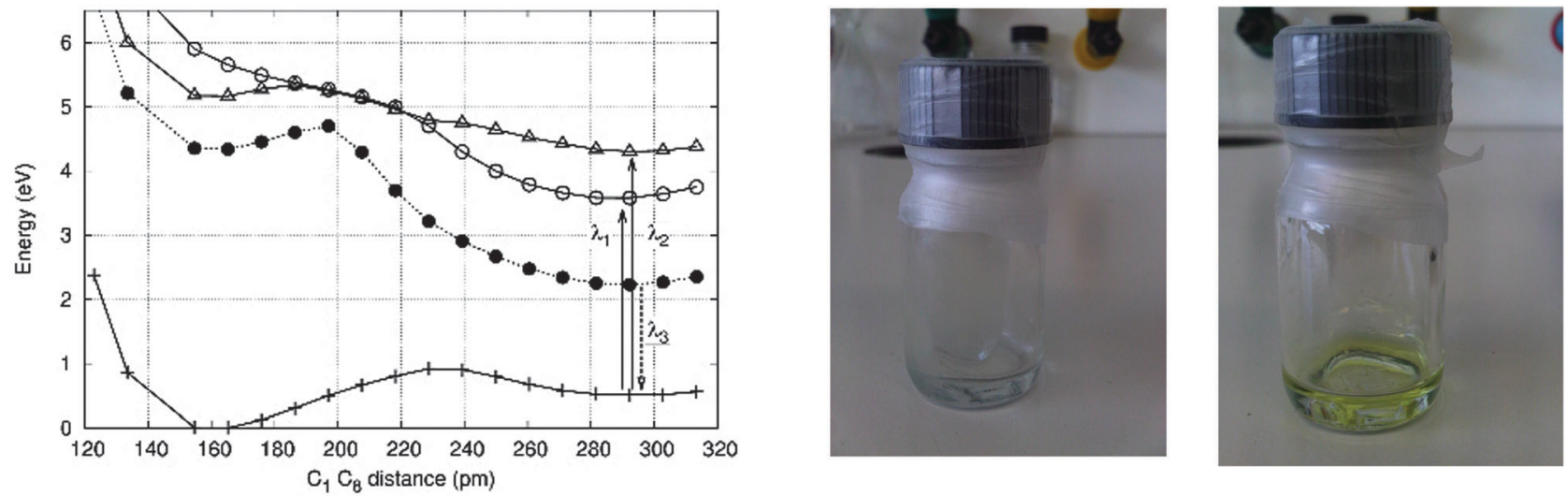

Fig. 3 Evolution of the CC2/aug-cc-pVDZ energy of the ground state $1^{1} \mathrm{~A}$ (solid line/cross symbols), the first triplet excited state $1^{3} \mathrm{~B}$ (dashed line/filled circles) and the two first singlet excited states (solid lines/empty circles $2^{1} B$ and empty triangles $2^{1} \mathrm{~A}$ ) along the breaking of the $\mathrm{C}_{1}-\mathrm{C}_{8}$ bond. Arrows in solid line styles at $290 \mathrm{pm}$ indicate the allowed electronic transitions at $\lambda_{1}=324 \mathrm{~nm}$ and $\lambda_{2}=394 \mathrm{~nm}$ which appear in the UV/visible spectrum and explain the colour of the compound. Arrow in dashed line indicates the emission transition at $\lambda_{3}=701 \mathrm{~nm}$ observed in the phosphorescence spectrum. Left picture: 1 at $-20^{\circ} \mathrm{C}$. Right picture: 1 at r.t. 


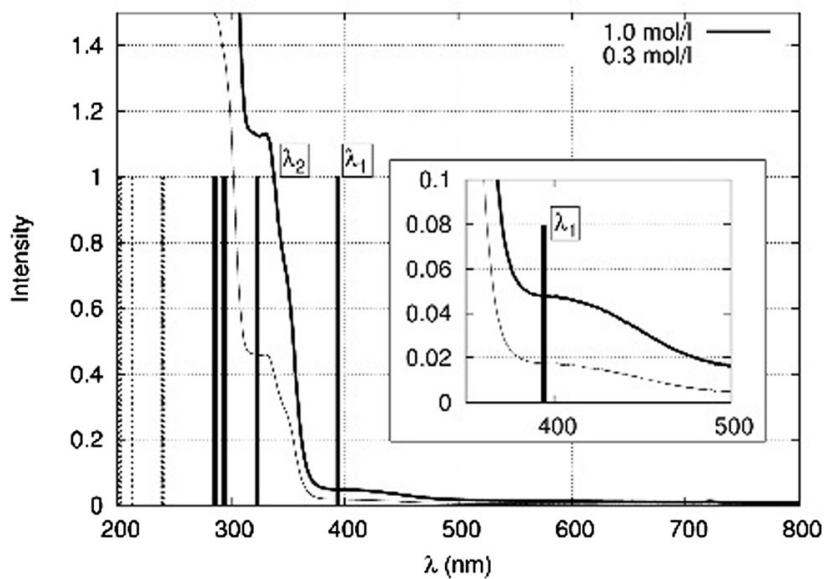

Fig. 4 UV/visible spectrum of 1 at two different concentrations (bold line: $1 \mathrm{~mol} \mathrm{l}^{-1}$, thin line; $0.33 \mathrm{~mol} \mathrm{l}^{-1}$ ). CC2/aug-cc-pVDZ calculations allowed transitions are reported as vertical lines with arbitrary intensities. The calculated absorptions of TMS-1 and TMS-7 are in dashed lines. The calculated absorptions of TMS- 9 are in solid lines. The wavelengths presented in Fig. 3 and 6 are reported $\left(\lambda_{1}=324 \mathrm{~nm}, \lambda_{2}=394 \mathrm{~nm}\right)$.

Table 1 First excitation energies and related wavelengths for TMS-1, TMS-7 and TMS-9

\begin{tabular}{lll}
\hline${ }^{(2 S+1)}$ Symmetry & $E($ ev $)$ & $\lambda(\mathrm{nm})$ \\
\hline $\mathrm{TMS}-1$ & & \\
${ }^{3} \mathrm{~B}$ & 4.34 & 286 \\
${ }^{3} \mathrm{~A}$ & 5.02 & 247 \\
${ }^{1} \mathrm{~A}$ & 5.19 & 239 \\
${ }^{1} \mathrm{~B}$ & 6.10 & 203 \\
${ }^{1} \mathrm{~B}$ & 6.27 & 198 \\
${ }^{1} \mathrm{~A}$ & 6.34 & 195 \\
& & \\
$\mathrm{TMS}-7$ & & 285 \\
${ }^{3} \mathrm{~B}$ & 4.35 & 250 \\
${ }^{3} \mathrm{~A}$ & 4.97 & 240 \\
${ }^{1} \mathrm{~A}$ & 5.17 & 213 \\
${ }^{1} \mathrm{~B}$ & 5.82 & 201 \\
${ }^{1} \mathrm{~A}$ & 6.18 & 199 \\
${ }^{1} \mathrm{~B}$ & 6.22 & \\
& & 701 \\
$\mathrm{TMS}-9$ & & 394 \\
${ }^{3} \mathrm{~B}$ & 1.77 & 324 \\
${ }^{1} \mathrm{~B}$ & 3.14 & 323 \\
${ }^{3} \mathrm{~A}$ & 3.82 & 294 \\
${ }^{1} \mathrm{~A}$ & 3.84 & 286 \\
${ }^{1} \mathrm{~A}$ & 4.22 & \\
${ }^{1} \mathrm{~B}$ & 4.33 &
\end{tabular}

in the range $630-730 \mathrm{~nm}$ with an optimal excitation wavelength of $312 \mathrm{~nm}$ (Fig. 5). This can be understood by a detailed inspection of the spectrum of the excited states of TMS-9 (Fig. 6). ${ }^{34}$ The $312 \mathrm{~nm}$ excitation corresponds to the $\mathrm{S}_{0}-\mathrm{S}_{2}$ transition. This transition is computed to be at $324 \mathrm{~nm}$ and is attributed to the shoulder at $330 \mathrm{~nm}$ in the UV spectrum. As $\mathrm{S}_{2}$ and $\mathrm{T}_{2}$ are quasi degenerated ( 3.82 vs. $3.84 \mathrm{eV}$ ), an inter system crossing (ISC) can occur between them. In a second step fluorescence occurs from $T_{2}$ to $T_{1}$. This latter transition is computed to be at $\lambda_{4}=603 \mathrm{~nm}$ and can be estimated experimentally by the energy difference between the energy of the $S_{2}$ state (at $330 \mathrm{~nm}$ ) and the energy of the $T_{1}$ state

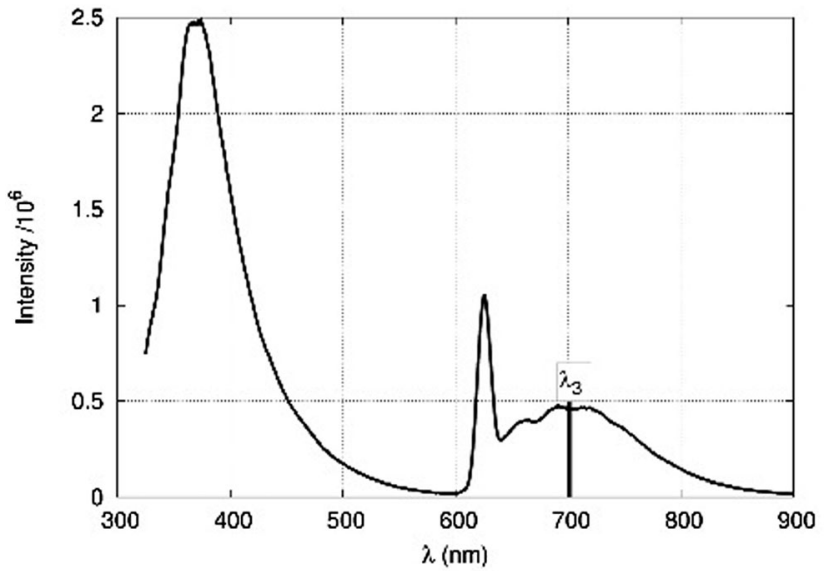

Fig. 5 Emission spectrum of 1 at $312 \mathrm{~nm}$. Emission at $350 \mathrm{~nm}$ is the fluorescence of the state populated by the excitation. The band at $624 \mathrm{~nm}$ is the harmonic of the $312 \mathrm{~nm}$ excitation. The phosphorescence emission band at $700 \mathrm{~nm}$ corresponds to the $\lambda_{3}$ CC2/aug-cc-pVDZ value of $701 \mathrm{~nm}$. Intensity of the calculated wavelength is arbitrary.

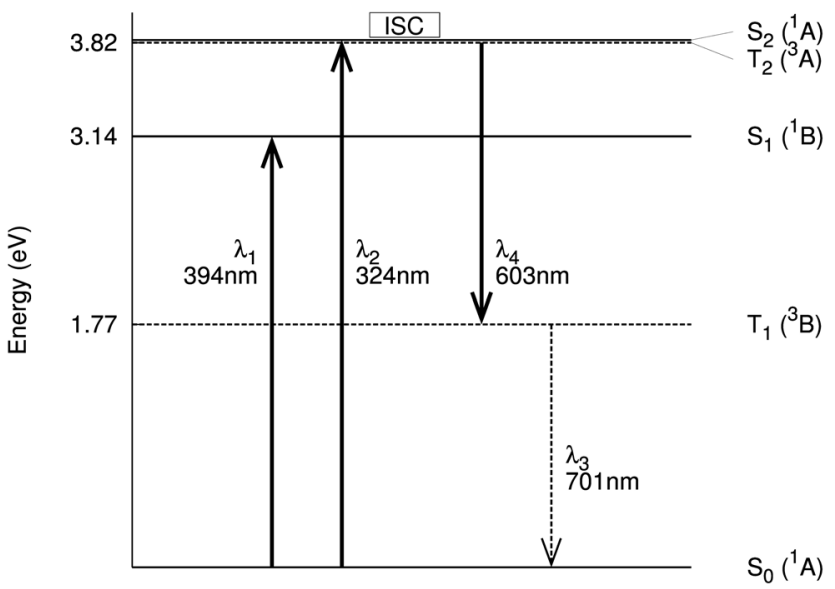

Fig. 6 Explanation of the electronic spectra of TMS-9 calculated at the CC2/aug-cc-pVDZ level of theory. Inter system crossing is likely to occur between $S_{2}$ and $T_{2}$. Solid lines are used for singlet states and allowed transitions (fluorescence). Dashed lines are used for triplet states and forbidden transitions (phosphorescence).

(630-730 nm emission). This gives a range for $\lambda_{4}$ of 602-693 nm. It should be emphasized that neither experimental nor theoretical evaluations of $\lambda_{4}$ take into account the geometry relaxation. They should then be considered as lower bound values. Thus, they appear to be in very good agreement. The broad emission at around $700 \mathrm{~nm}$ is the overlap of two transitions, the fluorescence from $T_{2}$ to $T_{1}$ and the phosphorescence from $T_{1}$ to $S_{0}$. We have shown that 9 exists despite its disfavoured electronic structure and the fact that it was not detected by spin trapping experiments. From this point we consider that it is the reactive species with $\mathrm{O}_{2}$.

2.3. Reaction of 9 with ${ }^{3} \Sigma_{\mathrm{g}} \mathrm{O}_{2}$. Two reaction pathways are possible: a chemical and a photochemical one. Both imply an inter system crossing (ISC), i.e. a jump from the triplet to the singlet potential energy surface, which is forbidden. Along the chemical pathway, ISC is likely to occur once $\mathrm{O}_{2}$ binds to 9 . 


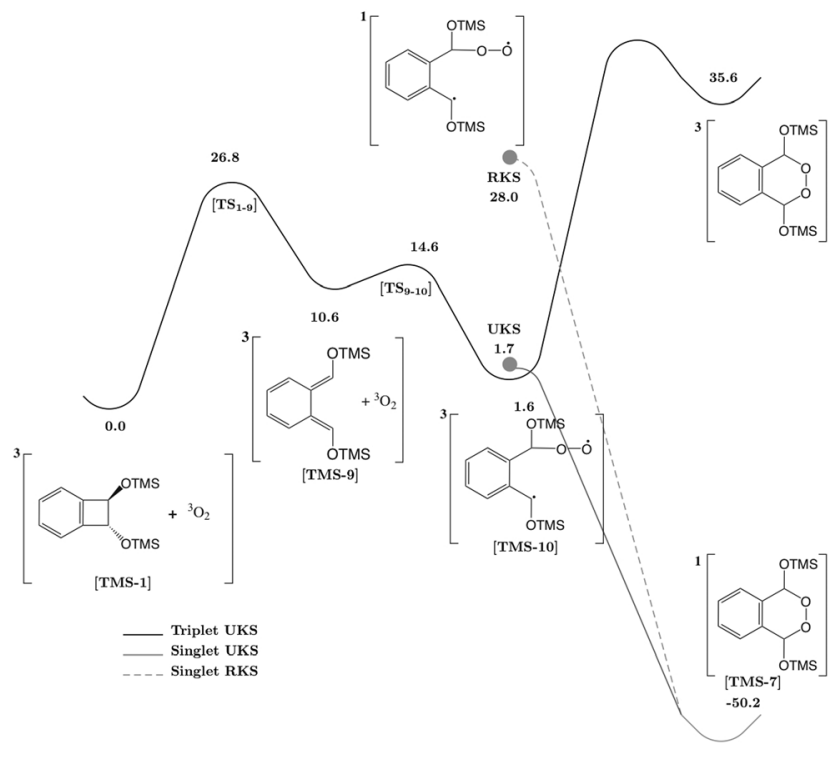

Fig. 7 Complete chemical reaction pathway. Energies relative to the separated reactants TMS-1 and $\mathrm{O}_{2}\left({ }^{3} \Sigma_{\mathrm{g}}\right)$ are in $\mathrm{kcal} \mathrm{mol}^{-1}$ at the PBEO/ def2-TZVP level of theory. Unrestricted and restricted Kohn-Sham formalism are compared because of spin contamination issues (see text).

The photochemical pathway starts from the first excited triplet state $\mathrm{T}_{1}$ of $\mathbf{9}$, which binds directly $\mathrm{O}_{2}$. In the following we calculate the two pathways.

2.3.a. Chemical pathway. The activation energy for the $\mathrm{O}_{2}\left({ }^{3} \Sigma_{\mathrm{g}}\right)$ approach is calculated to be $4.0 \mathrm{kcal} \mathrm{mol}^{-1}$ (Fig. 7). The exothermic reaction ( $9.0 \mathrm{kcal} \mathrm{mol}^{-1}$ from TMS-9) leads to the biradical TMS-10 that is a stable triplet molecule. The reaction cannot lead to TMS-7 on the triplet energy surface. Yet, experiment shows undeniably that 7 is the product of the reaction and that $\mathbf{1 0}$ exists in the medium as a biradical species. To go from 10 to 7 the system must undergo an ISC.

To invoke such a phenomenon the necessary conditions are that the triplet and singlet states of $\mathbf{1 0}$ have similar and quasidegenerated geometries. We shall now show that these conditions are fulfilled. We expect the singlet and triplet states to be quasi degenerated, as the atoms that formally carry a radical are spatially far from one another. On the optimized triplet geometry of TMS-10, a singlet state density was computed in both unrestricted and restricted Kohn-Sham formalism, respectively, denoted UKS and RKS. ${ }^{35}$

The restricted calculation leads to a very high energy of $28.0 \mathrm{kcal} \mathrm{mol}^{-1}$. This indicates that the RKS calculation converges to the first excited state of the molecule. The ground state could be modelled only via an unrestricted calculation, as the radical separation cannot be modelled with the doubly occupied orbital.

The energy cost to flip one electron from alpha to beta is the difference in energy between the triplet and the UKS singlet. It is found to be about $0.1 \mathrm{kcal} \mathrm{mol}^{-1}$. This energy difference is much lower than the precision of any DFT method. Our conclusion is that these two states of TMS-10, in the geometry of the triplet can be considered as degenerate. The singlet wave function is spin contaminated as expected. ${ }^{36}$

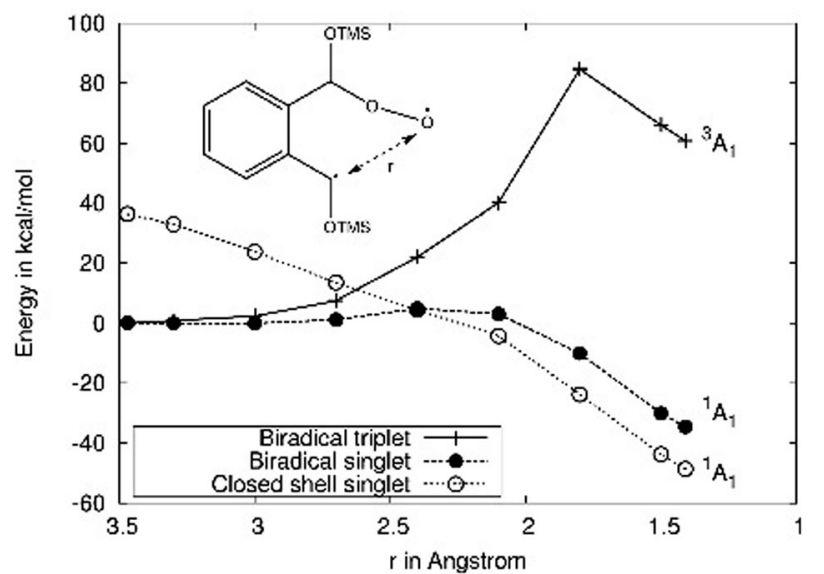

Fig. 8 CISD energy profile of the triplet and first two singlet states for the endo-peroxide TMS-7 formation.

Because spin contamination is an issue with DFT and to make sure that the low singlet state energy is not an artefact, CISD/def2-TZVP//PBE0/def2-TZVP calculations were performed along the geometrical deformation from TMS-10 to TMS-7 (Fig. 8). These are free of spin contamination. On each geometry, the ground state and the first singlet and triplet excited states were computed. The first point of Fig. 8 confirms the quasi degeneracy of the singlet and triplet states whereas the first excited singlet state is $38 \mathrm{kcal} \mathrm{mol}^{-1}$ higher. These results are in agreement with the DFT results and are completely reliable in terms of spin contamination. Along the $\mathrm{C}-\mathrm{O}$ bond formation, the triplet is destabilized by Pauli repulsion whereas the closed shell singlet state is stabilized until it becomes the ground state. For this process to occur, a small activation barrier, $4.8 \mathrm{kcal} \mathrm{mol}^{-1}$, was found at the CISD level.

At the DFT level of theory TMS-10 is not a minimum on the singlet energy surface: geometry optimizations converge to the singlet endo-peroxide form TMS-7 (activation barrier =

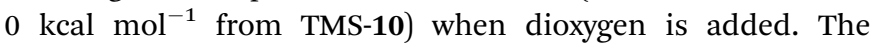
formation of TMS-7 in a singlet state is favoured energetically $\left(\right.$ reaction energy $\left.=-51.8 \mathrm{kcal} \mathrm{mol}^{-1}\right)$.

The DFT results confirmed by the CISD calculations show that the triplet state of TMS-10 is quasi degenerated with the singlet TMS-10. Thus, if there is enough energy available to cross the cyclobutene opening barrier (from $\mathbf{1}$ to $\mathbf{9}$ ) a chemical equilibrium takes place between $\mathbf{1}$ and $\mathbf{1 0}$ as soon as dioxygen is available. Experimentally, the energy is brought into the system by heating up at $40{ }^{\circ} \mathrm{C}$. Thus, 10 exists as a triplet state. As we have shown, the singlet and triplet states of $\mathbf{1 0}$ are quasi degenerated with similar geometries. The conditions for the inter system crossing are fulfilled. The system undergoes a formally forbidden triplet singlet transition, which leads irreversibly to 7 in an almost barrierless process.

The rate constants for the unimolecular and bimolecular reactions are calculated using the transition state theory. ${ }^{37}$ For the ring opening, from TMS-1 to TMS-9, a rate constant at $25{ }^{\circ} \mathrm{C}, k_{1}$ is $4.2 \times 10^{-9} \mathrm{~s}^{-1}\left(k_{-1}\right.$ is found to be $\left.1.6 \times 10^{-2} \mathrm{~s}^{-1}\right)$. The equilibrium constant of this reaction is $0.3 \times 10^{-6}$. At $40{ }^{\circ} \mathrm{C}$, the kinetic constant $k_{1}$ is ten times larger than the kinetic constant at $25{ }^{\circ} \mathrm{C}$. The rate constant of the bimolecular reaction 
which leads from TMS-9 to TMS-10 is $k_{2}=1.3 \times 10^{-3} \mathrm{~s}^{-1}$. The opposite unimolecular rate constant is $k_{-2}=5.5 \times 10^{-5} \mathrm{~s}^{-1}$. At $25{ }^{\circ} \mathrm{C}$, the concentration of 9 is small yet non-zero. If $\mathrm{O}_{2}\left({ }^{3} \Sigma_{\mathrm{g}}\right)$ is present in the medium, $\mathbf{1 0}$ is generated immediately which leads to the formation of 7 . The formation of $\mathbf{9}$ occurs until the reactant is consumed.

2.3.b. Photochemical pathway. As can be seen in Fig. 6 , in the $S_{1}$ state the molecule is not likely to undergo an inter system crossing because the triplet state $T_{1}$ is energetically too far from $S_{1}$. This is supported by the emission spectrum with an excitation wavelength of $400 \mathrm{~nm}$ in which only fluorescence is observed (emission spectrum at $400 \mathrm{~nm}$ is provided as $\mathrm{ESI} \dagger$ ). Thus the excited electronic state, which undergoes an ISC, is higher in energy. As was shown in the analysis of the UV/visible spectrum of $\mathbf{9}$, the $\mathrm{T}_{\mathbf{1}}$ state can be populated after an excitation from the ground state $\mathrm{S}_{0}$ to $\mathrm{S}_{2}$, an ISC from $S_{2}$ to $T_{2}$ (these two states being degenerated) and a fluorescence from $T_{2}$ to $T_{1}$. Even if unlikely, such ISC can occur in organic molecules as shown in the evaluation of elements of the spin-orbitcoupling matrix by Marian et al. ${ }^{38}$ and Russo et al. ${ }^{39}$ Once in the $\mathrm{T}_{1}$ state, 9 can react with $\mathrm{O}_{2}\left({ }^{3} \Sigma_{\mathrm{g}}\right)$ and leads to 7 with no activation energy. It is likely to be extremely fast, thus the only kinetically relevant step is the opening of the $\mathrm{C}_{4}$ ring of $\mathbf{9}$.

2.4. Conclusion on the reaction between 1 and $\mathrm{O}_{2}$. We have computed two reaction pathways for the reaction to lead to 7 from 1 and $\mathrm{O}_{2}$. Both pathways are likely to occur once the $\mathrm{C}_{4}$ ring of 1 opens to give 9. To distinguish the two postulated mechanisms, the oxidation reaction of 1 was carried out in the dark and inside an amberized NMR tube. After 5 hours at $40{ }^{\circ} \mathrm{C}$ in saturated $\mathrm{O}_{2} \mathrm{C}_{6} \mathrm{D}_{6}$ medium, the major product observed is endo-peroxide 7 with a conversion rate of $75 \%$. This experimental evidence allows us to conclude that the chemical pathway is certainly the one which occurred.

\section{Conclusions}

This work elucidated for the first time each step of the mechanism of spin-forbidden addition of triplet oxygen $\mathrm{O}_{2}\left({ }^{3} \Sigma_{\mathrm{g}}\right)$ to trans-1,2disiloxybenzocyclobutene 1 to give the corresponding endo-peroxide 7 by using both EPR/spin trapping and theoretical studies. First of all $\mathrm{EPR} /$ spin trapping proved that the auto-oxidation reaction of 1 could likely follow a radical pathway and the presence of free radical intermediates was undoubtedly proven. Theoretical studies have confirmed EPR/Spin trapping experiments and allow us to understand the formation of singlet state 7 . The required inter-crossing system for the forbidden reaction can take place through a chemical pathway (vs. a photochemical pathway) and between 10 and 7.

\section{Experimental section}

\section{Synthesis of compound 7}

To a stirred solution of 1 ( $30 \mathrm{mg}, 0.82 \mathrm{mmol})$ in $\mathrm{C}_{6} \mathrm{D}_{6}(1 \mathrm{~mL})$ was bubbled $\mathrm{O}_{2}$. The solution was then heated at $40{ }^{\circ} \mathrm{C}$ until the disappearance of the starting material (followed by ${ }^{1} \mathrm{H}$ NMR, 3 hours). Because of the instability of 7 during the purification step on silica gel, the crude reaction in $\mathrm{C}_{6} \mathrm{D}_{6}$ was used for NMR experiments. ${ }^{1} \mathrm{H}$ NMR $\left(300 \mathrm{MHz}, \mathrm{C}_{6} \mathrm{D}_{6}\right) \delta 0.18\left(6 \mathrm{H}, \mathrm{s}, 2 \times \mathrm{CH}_{3}\right)$, $0.20\left(6 \mathrm{H}, \mathrm{s}, 2 \times \mathrm{CH}_{3}\right), 0.98\left(18 \mathrm{H}, \mathrm{s}, 6 \times \mathrm{CH}_{3}\right), 6.16(2 \mathrm{H}, \mathrm{s}, 2 \times$ $\mathrm{CH})$, 7.07-7.09 (2H, m, $\left.2 \times \mathrm{CH}_{\mathrm{Ar}}\right), 7.27-7.29\left(2 \mathrm{H}, \mathrm{m}, 2 \times \mathrm{CH}_{\mathrm{Ar}}\right)$; ${ }^{13} \mathrm{C} \mathrm{NMR}\left(75 \mathrm{MHz}, \mathrm{C}_{6} \mathrm{D}_{6}\right) \delta-4.8\left(2 \times \mathrm{CH}_{3}\right),-3.9\left(2 \times \mathrm{CH}_{3}\right), 18.3$ $(2 \times \mathrm{C}), 259\left(6 \times \mathrm{CH}_{3}\right), 96.4(2 \times \mathrm{CH}), 125.4\left(2 \times \mathrm{CH}_{\mathrm{Ar}}\right), 128.4$ $\left(2 \times \mathrm{CH}_{\mathrm{Ar}}\right), 136.2\left(2 \times \mathrm{C}_{\mathrm{Ar}}\right) ; \mathrm{HRMS} \mathrm{m} / \mathrm{z}$ (ESI) calcd for $\mathrm{C}_{20} \mathrm{H}_{40} \mathrm{NO}_{4} \mathrm{Si}_{2}\left[\mathrm{M}+\mathrm{NH}_{4}\right]^{+}:$414.2490, found 414.2489.

\section{EPR/spin trapping experiments}

MNP was purchased from Sigma-Aldrich while DEPMPO was synthesized and purified according to methods previously described. ${ }^{14,40}$ The spin adducts were produced by mixing 1 (0.1-0.2 $\left.\mathrm{mol} \mathrm{L}^{-1}\right)$ and the spin trap $\left(0.2 \mathrm{~mol} \mathrm{~L}^{-1} \mathrm{MNP}\right.$ or $0.1 \mathrm{~mol} \mathrm{~L}^{-1}$ DEPMPO) in benzene. The system was allowed to react for $10 \mathrm{~min}^{-2} \mathrm{~h}$, and the sample was deoxygenated by argon bubbling before EPR analysis. The reaction medium was then transferred into a glass pipette closed with a septum for EPR analysis. Blank tests were also performed in the absence of $\mathrm{O}_{2}$, of 1 or of the spin trap. EPR spectra were recorded at room temperature $\left(20-22{ }^{\circ} \mathrm{C}\right)$ on a continuous wave X-band Bruker EMX spectrometer. The following conditions were used: modulation frequency, $100 \mathrm{kHz}$; non saturating microwave power, $20 \mathrm{~mW}$; modulation amplitude, 0.1-0.15 mT; receiver gain, $10^{5}-10^{6}$; time constant, 1.28-655 ms; scan time, 60-180 s. The hyperfine coupling constants $a_{\mathrm{x}}$ were determined after simulation of the experimental EPR spectra using the WinSim2002 software. ${ }^{15,41}$

\section{Notes and references}

1 (a) S. Inack-Ngi, K. Cherry, V. Heran, L. Commeiras, J.-L. Parrain, A. Duchene, M. Abarbri and J. Thibonnet, Chem.-Eur. J., 2011, 17, 13692; (b) S. Inack-Ngi, R. Rahmani, L. Commeiras, G. Chouraqui, J. Thibonnet, A. Duchene, M. Abarbri and J.-L. Parrain, Adv. Synth. Catal., 2009, 351, 779; (c) A. Duchene, J. Thibonnet, J.-L. Parrain, E. Anselmi and M. Abarbri, Synthesis, 2007, 597; (d) S. Rousset, M. Abarbri, J. Thibonnet, J.-L. Parrain and A. Duchene, Tetrahedron Lett., 2003, 44, 7633; (e) S. Rousset, J. Thibonnet, M. Abarbri, A. Duchene and J.-L. Parrain, Synlett, 2000, 260; $(f)$ S. Rousset, M. Abarbri, J. Thibonnet, A. Duchene and J.-L. Parrain, Org. Lett., 1999, 1, 701.

2 (a) A. Bartoli, F. Rodier, L. Commeiras, J.-L. Parrain and G. Chouraqui, Nat. Prod. Rep., 2011, 28, 763; (b) A. Bartoli, G. Chouraqui and J.-L. Parrain, Org. Lett., 2012, 14, 122; (c) F. Rodier, M. Rajzmann, J.-L. Parrain, G. Chouraqui and L. Commeiras, Chem.-Eur. J., 2013, 19, 2467.

3 R. Blanc, V. Heran, R. Rahmani, L. Commeiras and J.-L. Parrain, Org. Biomol. Chem., 2010, 8, 5490.

4 S. Dubois, F. Rodier, R. Blanc, R. Rahmani, V. Heran, J. Thibonnet, L. Commeiras and J.-L. Parrain, Org. Biomol. Chem., 2012, 10, 4712.

5 (a) K. Yamamoto, M. F. Hentemann, J. G. Allen and S. J. Danishefsky, Chem.-Eur. J., 2003, 9, 3242; (b) J. Barluenga, F. Aznar and M. A. Palomero, Chem.-Eur. J., 2002, 8, 4149; (c) J. Barluenga, M. A. Fernandez-Rodriguez and E. Aguilar, Org. Lett., 2002, 4, 3659; (d) J. G. Allen and S. J. Danishefsky, 
J. Am. Chem. Soc., 2001, 123, 351; (e) M. F. Hentemann, J. G. Allen and S. J. Danishefsky, Angew. Chem., Int. Ed., 2000, 39, 1937; $(f)$ J. G. Allen, M. F. Hentemann and S. J. Danishefsky, J. Am. Chem. Soc., 2000, 122, 571.

6 All supporting information (ref. 5) neither mentioned the use of degassed solvent.

7 W. R. Roth, T. Ebbrecht and A. Beitat, Chem. Ber., 1988, 121, 1357.

8 C. Evans, Aldrichimica Acta, 1979, 12, 23.

9 B. F. Minaev, N. A. Murugan and H. Ågren, Int. J. Quantum Chem., 2013, 113, 1847-1867.

10 (a) B. F. Minaev and H. Ågren, THEOCHEM, 1998, 434, 193-206; (b) B. F. Minaev and H. Ågren, EPA Newsl., 1999, 65, 7-37.

11 See ESI $\dagger$ for EPR spectrum.

12 Nitroso spin traps have an important advantage over nitrones in that the addend is directly bound to the nitrogen, thereby yielding additional hyperfine couplings. Therefore, they are the traps of choice to differentiate radicals centered on a primary, a secondary or a tertiary carbon.

13 M. Perkins and B. Roberts, J. Chem. Soc., Perkin Trans. 1, 1974, 297.

14 E. Janzen, D. Lindsay and D. Haire, J. Am. Chem. Soc., 1990, 112, 8279.

15 The free WinSim program is available at the following address: www.niehs.nih.gov/research/resources/software/ tox-pharm/tools/index.cfm.

16 (a) T. Wajer, T. Mackor, T. de Boer and J. van Voorst, Tetrahedron, 1967, 23, 4021; (b) K. Makino, N. Suzuki, F. Moriya, S. Rokushida and H. Hatano, Anal. Lett., 1980, 13, 311; (c) G. R. Buettner, Free Radical Biol. Med., 1987, 3, 259.

17 (a) C. Fréjaville, H. Karoui, B. Tuccio, F. Le Moigne, M. Culcasi, S. Pietri, R. Lauricella and P. Tordo, J. Med. Chem., 1995, 38, 258; (b) B. Tuccio, R. Lauricella, C. Fréjaville, J.-C. Bouteiller and P. Tordo, J. Chem. Soc., Perkin Trans. 2, 1995, 295; (c) S. Dikalov, P. Tordo, A. Motten and R. Mason, Free Radical Biol. Med., 2003, 37, 705.

18 (a) S. Barbati, J.-L. Clément, G. Olive, V. Roubaud, B. Tuccio and P. Tordo, in NATO ASI Series, Serie A* Life Sciences, ed. F. Minisci and Kluwer Academic Publishers, Dordrecht, 1997, vol. 27, p. 39; (b) H. Karoui, F. Chalier, J.-P. Finet and P. Tordo, Org. Biomol. Chem., 2011, 9, 2473.

19 T. K. Kemp, Prog. React. Kinet. Mech., 1999, 24, 287.

20 M. Triquigneaux, L. Charles, C. André-Barrès and B. Tuccio, Org. Biomol. Chem., 2010, 8, 1361.

21 C. Adamo and V. J. Barone, Chem. Phys., 1999, 110, 6158.

22 F. Weigend, M. Haser, H. Patzelt and R. Ahlrichs, Chem. Phys. Lett., 1998, 294, 143.

23 F. Weigend and R. Ahlrichs, Phys. Chem. Chem. Phys., 2005, 7, 3297.

24 (a) O. Christiansen, H. Koch and P. Jørgensen, Chem. Phys. Lett., 1995, 243, 409; (b) C. Hättig and F. Weigend, J. Chem. Phys., 2000, 113, 5154; (c) C. Hättig and A. Köhn, J. Chem. Phys., 2002, 117, 6939; (d) C. Hättig and K. Hald, Phys. Chem. Chem. Phys., 2002, 4, 2111; (e) C. Hättig, A. Köhn and
K. Hald, J. Chem. Phys., 2002, 116, 5401; $(f)$ C. Hättig, A. Hellweg and A. Köhn, Phys. Chem. Chem. Phys., 2006, 8, 1159.

25 (a) T. H. Dunning Jr., J. Chem. Phys., 1989, 90, 1007-1023; (b) D. E. Woon and T. H. Dunning, Jr., J. Chem. Phys., 1993, 98, 1358.

26 F. Weigend, A. Köhn and C. Hättig, J. Chem. Phys., 2001, 116, 3175.

27 P. J. Knowles and H. J. Werner, Chem. Phys. Lett., 1988, $145,514$.

28 H. J. Werner and P. J. Knowles, J. Chem. Phys., 1988, 89, 5803.

29 TURBOMOLE V6.3 2011, a development of University of Karlsruhe and Forschungszentrum Karlsruhe $\mathrm{GmbH}$, 1989-2007, TURBOMOLE GmbH, since 2007; available from http://www.turbomole.com.

30 MOLPRO, version 2008.1, a package of ab initio programs, H.-J. Werner, P. J. Knowles, R. Lindh, F. R. Manby, M. Schutz et al., see http://www.molpro.net.

31 K. L. Schuchardt, B. T. Didier, T. Elsethagen, L. S. Sun, V. Gurumoorthi, J. Chase, J. Li and T. L. Windus, J. Chem. Inf. Model., 2007, 47, 1045.

32 Y. Tantirungrotechai, K. Phanasant, S. Roddecha, P. Surawatanawong, V. Sutthikhum and J. Limtrakul, THEOCHEM, 2006, 760, 189.

33 (a) W. Choy and H. Yang, J. Org. Chem., 1988, 53, 5796; (b) M. F. Hentemann, J. G. Allen and S. J. Danishefsky, Angew. Chem., Int. Ed., 2000, 39, 1937.

34 Electronic states are labelled using both spectroscopic ${ }^{(2 \mathrm{~S}+1)} \Gamma$ or Jablonski notation (S: singlet, $\mathrm{T}$ : triplet, index: 0 for ground state and larger or equal to 1 for excited states). As TMS-9 has a $C_{2}$ symmetry values A and B are possible for the irreducible representations $\Gamma$ according to the symmetry of the state.

35 IUPAC, Compendium of Chemical Terminology, compiled by A. D. McNaught and A. Wilkinson, Blackwell Scientific Publications, Oxford, 2nd edn (the "Gold Book”), 1997, XML on-line corrected version: http://goldbook.iupac.org (2006-) created by M. Nic, J. Jirat, B. Kosata; updates compiled by A. Jenkins, ISBN 0-9678550-9-8, DOI: 10.1351/ goldbook.

36 J. A. Pople, P. M. W. Gill and N. C. Handy, Int. J. Quantum Chem., 1995, 56, 303.

37 J. I. Steinfeld, J. S. Francisco and W. L. Hase, Chemical Kinetics Dynamics, Prentice Hall, Upper Saddle River, New Jersey, 1998.

38 J. Tachen, H. Gilka and C. M. Marian, Phys. Chem. Chem. Phys., 2007, 9, 5209-5221.

39 (a) A. D. Quartarrolo, S. G. Chiodo and N. Russo, J. Chem. Theory Comput., 2010, 6, 3176-3189; (b) A. D. Quartarrolo, S. G. Chiodo and N. Russo, J. Comput. Chem., 2012, 33, 1091-1100.

40 S. Barbati, J.-L. Clément, C. Fréjaville, J.-C. Bouteiller, P. Tordo and J.-C. Yadan, Synthesis, 1999, 2036.

41 D. R. Duling, J. Magn. Reson., Ser. B, 1994, 104, 105. 